\title{
Characterization of a minimal pKW2124 replicon from Weissella cibaria KLC140 and its application for the construction of the Weissella expression vector pKUCm1
}

\author{
Hye-Jin Ku' ${ }^{1}$, Myeong Soo Park ${ }^{2}$ and Ju-Hoon Lee ${ }^{1 *}$ \\ ' Department of Food Science and Biotechnology, Institute of Life Sciences and Resources, Kyung Hee University, Yongin, South Korea \\ ${ }^{2}$ Department of Hotel Culinary Arts, Yeonsung University, Anyang, South Korea
}

\author{
Edited by: \\ Vincenzina Fusco, National Research \\ Council of Italy - Institute of Sciences \\ of Food Production, Italy

\section{Reviewed by:} \\ Eric Altermann, AgResearch Ltd., \\ New Zealand \\ Giuseppe Blaiotta, Università degli \\ Studi di Napoli Federico II, Italy

\section{*Correspondence:} \\ Ju-Hoon Lee, Department of Food \\ Science and Biotechnology, Institute \\ of Life Sciences and Resources, \\ Kyung Hee University, Yongin 446-701, \\ South Korea \\ e-mail: juhlee@khu.ac.kr
}

A 2.1-kb plasmid was previously isolated from Weissella cibaria KLC140 in kimchi and cloned into pUC19 along with the s/pA and gfp genes, resulting in an 8.6-kb pKWCSLGFP construct for use as a novel surface display vector. To reduce the size of the vector, the minimal replicon of pKW2124 was determined. The pKW2124 plasmid contains a putative origin of replication (ori), a potential ribosomal binding site (RBS), and the repA gene encoding a plasmid replication protein. To conduct the minimal replicon experiment, four different PCR products (MR1, ori+RBS+repA; MR2, RBS+repA; MR2', repA; MR3, fragment of repA) were obtained and cloned into pUC19 (pKUCm1, pKUCm2, pKUCm2', and pKUCm3, respectively) containing the chloramphenicol acetyltransferase (CAT) gene. These constructed vectors were electroporated into W. confusa ATCC 10881 with different transformation efficiencies of $1.5 \times 10^{5} \mathrm{CFU} / \mu \mathrm{g}, 1.3 \times 10^{1} \mathrm{CFU} / \mu \mathrm{g}$, and no transformation, respectively, suggesting that the putative ori, RBS, and repA gene are essential for optimum plasmid replication. Subsequent segregational plasmid stability testing of pKUCm1 and pKUCm2 showed that the vector pKUCm1 is highly stable up to 100 generations but pKUCm2 was completely lost after 60 generations, suggesting that the putative ori may be important for plasmid stability in the host strain. In addition, a host range test of pKUCm1 revealed that it has a broad host range spectrum including Weissella, Lactococcus, Leuconostoc, and even Lactobacillus. To verify the application of pKUCm1, the $\beta$-galactosidase gene and its promoter region from $W$. cibaria KSD1 were cloned in the vector, resulting in pKUGal. Expression of the $\beta$-galactosidase gene was confirmed using blue-white screening after IPTG induction. The small and stable pKUGal vector will be useful for gene transfer, expression, and manipulation in the Weissella genome and in other lactic acid bacteria.

Keywords: Weissella, minimal replicon, expression vector, $\beta$-galactosidase, theta replication

\section{INTRODUCTION}

Members of the genus Weissella are Gram-positive, non-spore forming, heterofermentative, non-motile, and short-rod bacteria belonging to the family Leuconostocaceae (Collins et al., 1993; Lee et al., 2002). Since Weissella was first characterized by Collins etal. (1993), this novel species has been continuously isolated from various sources including fermented foods (Lee et al., 2002; Magnusson et al., 2002; Tohno et al., 2013). To date, 14 species of Weissella are listed in the Taxonomic Outline of Bacteria and Archaea, release 7.7 (TOBA 7.7; Garrity et al., 2007). They are recognized as lactic acid bacteria (LAB) and generally produce lactic acid and various exopolysaccharides (EPSs), which are likely involved in food flavor and texture (Galle et al., 2010). Interestingly, novel bacteriocins (weissellicin 110 from W. cibaria, and weissellicin $\mathrm{Y}$ and $\mathrm{M}$ from $W$. hellenica) were reported in these strains, suggesting the potential for new food preservatives (Srionnual et al., 2007; Masuda et al., 2012). Furthermore, the $W$. confusa strain PL9001 inhibited Helicobacter pylori, suggesting that Weissella has the potential as a probiotic for promoting stomach health (Nam et al., 2002).
To explore the host beneficial effects of Weissella, its molecular and genomic study is required. Since the draft genome sequence of W. cibaria KACC 11862 was first reported in 2011 (Kim et al., 2011), the complete genome sequence of W. koreensis KACC 15510 (Lee et al., 2011) and the draft genome sequences of W. thailandensis fsh4-2 (Benomar etal., 2011), W. koreensis KCTC $3621^{\mathrm{T}}$ (Lee et al., 2012), and W. confusa LBAE C39-2 (Amari et al., 2012) were reported and deposited in the GenBank database. However, only a few Weissella plasmids were sequenced to develop molecular tools to study their genomes, including pKLCA (1.5-kb), pKLCB (3.4-kb), pKLCC (10.0-kb), and pKW2124 (2.1-kb) from W. cibaria KLC140 (Park et al., 2007; Kim et al., 2013). In an effort to develop the first Weissella cloning and expression vector system, the 2.1-kb theta-replicating plasmid pKW2124 was characterized (Kim et al., 2013). To construct a Weissella cloning/expression vector, the pTSLGFP vector containing a fused slpA gene encoding a surface layer protein with the $g f p$ gene was cloned into pKUWCAT containing the entire pKW2124 plasmid and the chloramphenicol acetyltransferase (CAT) gene, resulting in a $8.6-\mathrm{kb}$ pKWCSLGFP vector (Kim et al., 2013). However, minimization 
of the vector is needed, via determination of the minimal replicon of pKW2124, to increase transformation efficiency and vector stability.

In this study, the small plasmid from W. cibaria KLC140, pKW2124, was dissected and tested to determine the minimal replicon. Using the minimal pKW2124 replicon, the stable Escherichia coli-Weissella shuttle cloning vector $\mathrm{pKUCm} 1$ was constructed and its host spectrum was studied. This minimalized expression vector was used for the cloning and heterologous expression of the $\beta$-galactosidase gene (pKUGal), indicating that this improved expression vector system will be a useful molecular tool for gene cloning and expression in Weissella.

\section{MATERIALS AND METHODS BACTERIAL STRAINS, PLASMIDS, PCR PRIMERS, AND GROWTH CONDITIONS}

The bacterial strains, plasmids, and primers used in this study are listed in Table 1. All Weissella species were incubated anaerobically at $37^{\circ} \mathrm{C}$ in de Man-Rogosa-Sharpe (MRS) medium (Difco,

Table 1 | Bacterial strains, plasmids, and primers used in this study.

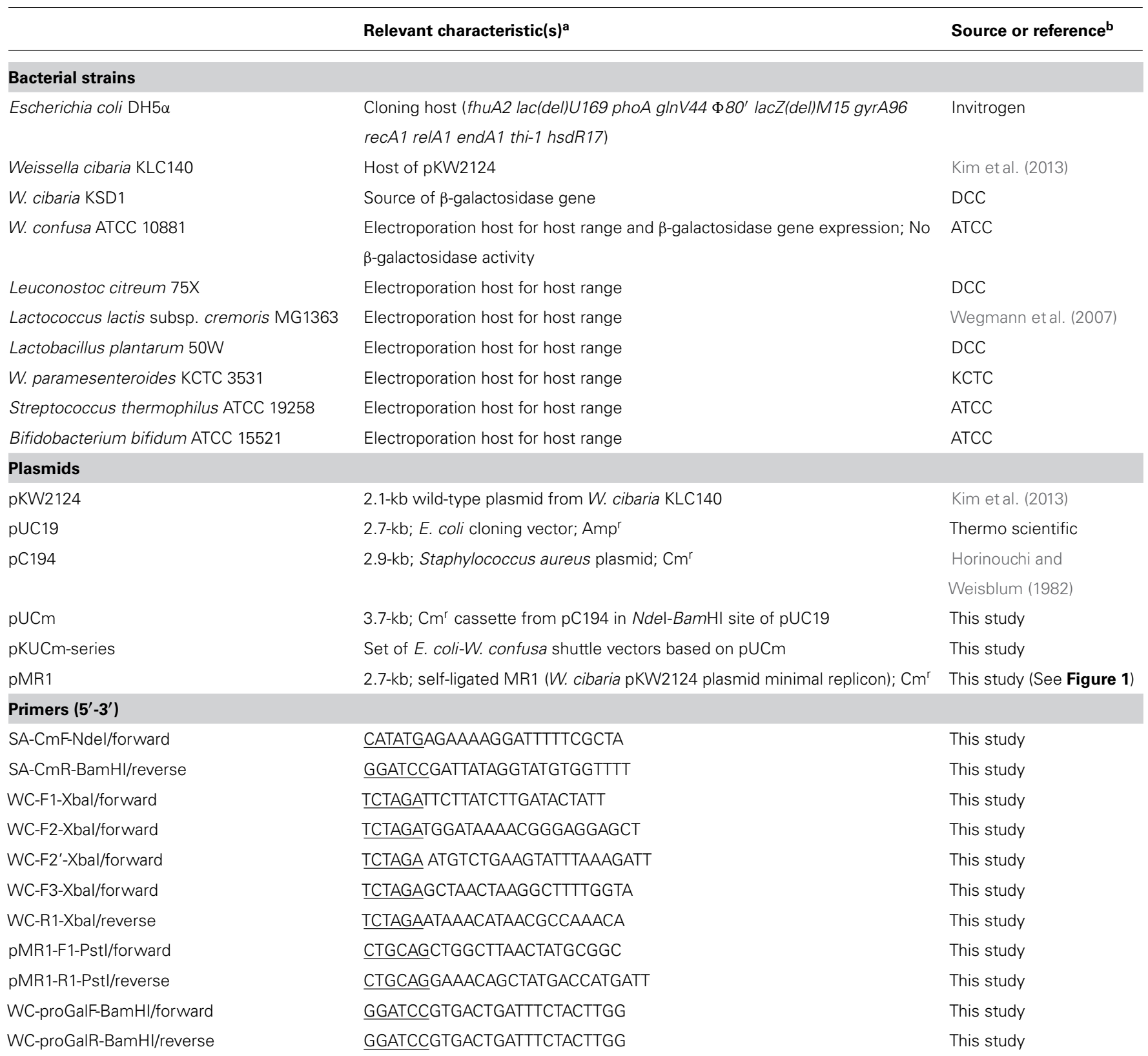

${ }^{a} A m p^{r}$ and $\mathrm{Cm}^{r}$, resistance to ampicillin and chloramphenicol, respectively; Underlined sequence in primers indicate introduced restriction enzyme sites (Ndel, BamHI, Xbal, and Pstl).

${ }^{b}$ ATCC, American Type Culture Collection; KCTC, Korean Collection for Type Culture; DCC, Dairy Culture Collection, Kyung Hee University. 
A
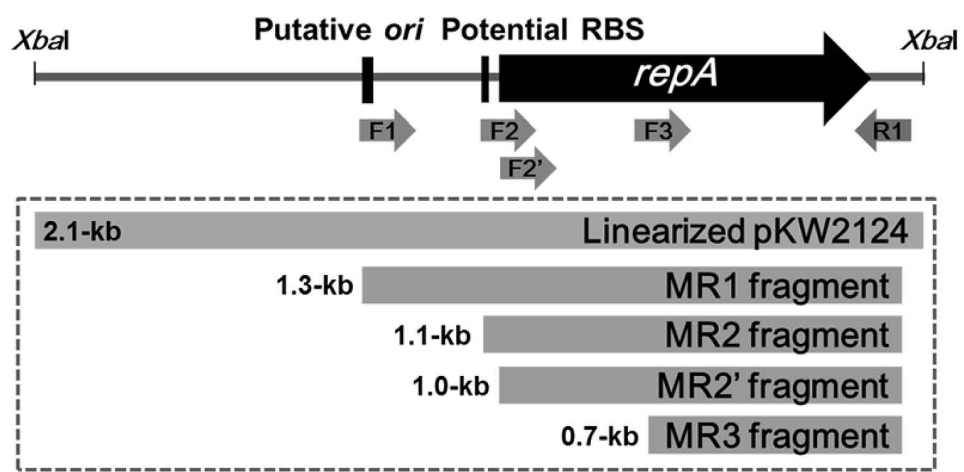

B

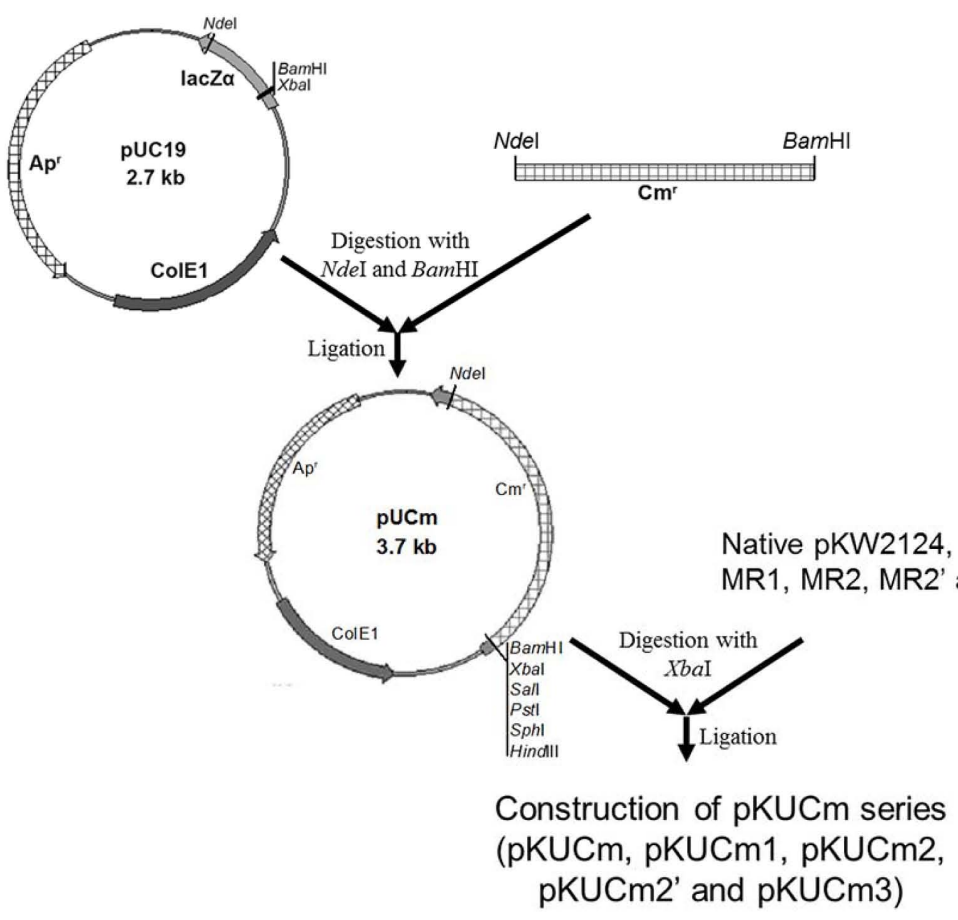

FIGURE 1 | Preparation of PCR-amplified potential Weissella minimal replicons and their cloning into pUCm vector. (A) Four different PCR products (MR1, MR2, MR2', and MR3) were obtained to determine the minimal replicon of pKW2124. (B) The entire pKW2124, MR1, MR2, MR2', and MR3 products were cloned into $\mathrm{pUCm}$ (pUC19+CAT gene), resulting in pKUCm, pKUCm1, pKUCm2, pKUCm2', and pKUCm3, respectively. F1, F2, F2', F3, and R1 indicate the WC-F1-Xbal, WC-F2-Xbal, WC-F2'-Xbal, WC-F3-Xbal, and WC-R1-Xbal PCR primers, respectively.
Detroit, MI, USA) and E. coli DH5 $\alpha$ (Invitrogen, Carlsbad, CA, USA) was grown with shaking in Luria-Bertani (LB) medium (Difco) at $37^{\circ} \mathrm{C}$. Ampicillin sulfate (Sigma, St. Louis, MO, USA) was added to the E. coli growth medium for selection at $50 \mu \mathrm{g} / \mathrm{ml}$. Weissella and other electroporation host bacteria were selected using chloramphenicol (USB Corporation, Santa Clara, CA, USA) with the following appropriate concentrations, $6.0 \mu \mathrm{g} / \mathrm{ml}$ for Weissella, Lactobacillus and Bifidobacterium, $5.0 \mu \mathrm{g} / \mathrm{ml}$ for Lactococcus and Leuconostoc, and $3.0 \mu \mathrm{g} / \mathrm{ml}$ for Streptococcus. Agar medium was prepared with supplementation of $1.8 \%$ Bacto agar (Difco).

\section{PLASMID PREPARATION AND MOLECULAR TECHNIOUES}

Plasmid preparation from all Weissella species was followed as previously described by O'Sullivan and Klaenhammer (1993). For plasmid preparation, bacterial cells of $15 \mathrm{ml}$ overnight culture was harvested and resuspended in $1 \mathrm{ml}$ of TES buffer $(30 \mathrm{mM}$ Tris $\mathrm{HCl}, \mathrm{pH}$ 8.0, $50 \mathrm{mM} \mathrm{NaCl}, 5 \mathrm{mM}$ EDTA, pH 8.0, final concentration). After pelleting, the cells were resuspended in $200 \mu \mathrm{l}$ of sucrose-lysozyme solution $(30 \mathrm{mg} / \mathrm{ml}$ lysozyme in $25 \%$ sucrose, $50 \mathrm{mM}$ Tris $\mathrm{HCl}, \mathrm{pH} 8.0$, and $1 \mathrm{mM}$ EDTA, pH 8.0, final concentration) and were incubated for $1 \mathrm{~h}$ at $37^{\circ} \mathrm{C}$. After treatment with alkaline sodium dodecyl sulfate (SDS) solution (3\% SDS in $0.2 \mathrm{~N}$ 
$\mathrm{NaOH}$, final concentration) for $7 \mathrm{~min}$ at room temperature, $300 \mu \mathrm{l}$ of pre-chilled $3 \mathrm{M}$ sodium acetate ( $\mathrm{pH} 4.8$ ) was added and gently mixed. After centrifugation, the supernatant was mixed with a $650 \mu \mathrm{l}$ of isopropanol and centrifuged to pellet plasmid DNA. The DNA pellet was resuspended in $320 \mu \mathrm{l}$ of molecular-grade water $(\mathrm{MW})$ with $2 \mu \mathrm{l}$ of RNase A $(100 \mathrm{mg} / \mathrm{ml})$ and incubated for $1 \mathrm{~h}$ at $37^{\circ} \mathrm{C}$. After RNase A treatment, the plasmid DNA was extracted using saturated phenol-chloroform/isoamyl alcohol and the water layer was transferred into sterilized $1.5 \mathrm{ml}$ tube. The plasmid DNA was precipitated with ice-cold ethanol and resuspended with $20 \mu \mathrm{l}$ of MW. Plasmid DNA from E. coli was isolated using the Axyprep Plasmid Miniprep Kit (Axygen Biosciences, Union City, CA, USA) according to the manufacturer's instructions. The general procedures for restriction enzyme digestion and recombinant DNA preparation were carried out according to standard procedures (Sambrook et al., 2001). All PCR reactions were performed with KOD -Plus- high fidelity DNA polymerase (TOYOBO, Osaka, Japan) according to the manufacturer's protocols.

\section{PLASMID TRANSFER}

Escherichia coli $\mathrm{DH} 5 \alpha$ (Invitrogen) cells were used as the competent strain for standard heat-shock transformation (Sambrook et al., 2001). The electroporation procedure and conditions for $W$. confusa ATCC 10881 are as follows. An overnight MRS broth culture of W. confusa ATCC 10881 was sub-inoculated in MRS broth containing $0.25 \mathrm{M}$ sucrose and grown to an optical density of 0.5 at $600 \mathrm{~nm}$. The cells were harvested by centrifugation and washed twice by re-suspension of the pellet with the same volume of icecold electroporation buffer ( $0.5 \mathrm{M}$ sucrose and $10 \%$ glycerol). Then, the pellet was re-suspended in ice-cold electroporation buffer containing lysozyme (600 $\mathrm{U} \mathrm{ml}^{-1}$, final concentration) and incubated at $37^{\circ} \mathrm{C}$ for $20 \mathrm{~min}$. Cells were washed twice with ice-cold electroporation buffer and re-suspended in the small volume of buffer ( $1 \%$ of total culture volume). For electroporation, $50 \mu \mathrm{l}$ of competent cells and $0.1 \mu \mathrm{g}$ plasmid DNA were electroporated in a $2-\mathrm{mm}$ pre-chilled cuvette at $25 \mu \mathrm{F}, 400 \Omega$, and $2 \mathrm{kV} \mathrm{cm}^{-1}$. Cells were immediately re-suspended in $1.0 \mathrm{ml}$ recovery medium (MRS broth supplemented with $0.5 \mathrm{M}$ sucrose) and incubated at $37^{\circ} \mathrm{C}$ for $2 \mathrm{~h}$. Transformants were selected using MRS agar containing $6.0 \mu \mathrm{g} / \mathrm{ml}$ chloramphenicol for 2 days at $37^{\circ} \mathrm{C}$.

\section{SEGREGATIONAL STABILITY OF THE MINIMAL REPLICON}

The segregational stability of the pKUCm series of plasmids harbored in W. confusa ATCC 10881 without antibiotic selection was monitored as described previously (Lee and O'Sullivan, 2006). The presence of plasmids was finally verified by plasmid preparations and subsequent restriction enzyme digestion.

\section{DETERMINATION OF pKUCm1 HOST RANGE}

The electroporation host strains listed in Table $\mathbf{1}$ were used for transformation with pKUCml and the colonies were selected against appropriate chloramphenicol concentrations. The selected colonies were used for plasmid re-isolation and restriction enzyme digestion for confirmation of the pKUCm1 host range.

\section{$\beta$-GALACTOSIDASE GENE CLONING, EXPRESSION, AND ASSAYS FOR ACTIVITY}

The promoter region of the $\beta$-galactosidase gene was predicted using the BPROM program (Li, 2011). The $\beta$-galactosidase gene and its promoter region (ProGal) in W. cibaria KSD1 were PCR amplified with primers WC-proGalF-BamHI and WC-proGalRBamHI (Table 1) using genomic DNA from $W$. cibaria KSD1 as a template. The PCR conditions were as follows: $94^{\circ} \mathrm{C}$ for $2 \mathrm{~min}$ followed by 30 cycles of $15 \mathrm{~s}$ at $94^{\circ} \mathrm{C}, 30 \mathrm{~s}$ at $52^{\circ} \mathrm{C}, 3 \mathrm{~min}$ at $68^{\circ} \mathrm{C}$, and a final extension for $5 \mathrm{~min}$ at $68^{\circ} \mathrm{C}$. The PCR product was purified, digested with $\mathrm{BamHI}$, and cloned into the BamHI site of pKUCm1, resulting in pKUGal. The pKUGal plasmid was electroporated into W. confusa ATCC 10881 and $\beta$-galactosidase activity was confirmed by blue-white screening on MRS agar plates supplemented with $6.0 \mu \mathrm{g} / \mathrm{ml}$ chloramphenicol, $50 \mu \mathrm{l} 20 \mathrm{mg} / \mathrm{ml}$ X-gal (Bio Basic, Amherst, NY, USA), and $10 \mu$ l 1.0 M IPTG (Bio Basic). Furthermore, $\beta$-galactosidase activity was measured using Miller's (1972) method. One unit of $\beta$-galactosidase activity was defined as the amount of enzyme required to produce $1.0 \mu \mathrm{mol}$ of o-nitrophenol per min.

\section{RESULTS \\ CONSTRUCTION OF pKW2124-DERIVED VECTORS}

The entire pKW2124 plasmid sequence contains a plasmid replication cluster consisting of a putative origin of replication (ori), a potential ribosomal binding site (RBS), and a repA gene encoding a plasmid replication protein (Kim et al., 2013). To elucidate their functions and to determine the minimal replicon of pKW2124, four different PCR amplicons of pKW2124 (MR1, ori+RBS+repA; MR2, RBS+repA; MR2', rеpA; MR3, fragment of $r e p A)$ were prepared with different oligonucleotide primer sets containing $X b a \mathrm{I}$ for cloning (Figure 1A). For efficient selection of grampositive bacterial transformants, the $1.3-\mathrm{kb}$ cat gene from the Staphylococcus aureus plasmid pC194 was introduced into the NdeI-BamHI sites of pUC19, resulting in the 3.7-kb vector, pUCm. A linearized whole plasmid pKW2124 by XbaI restriction and each PCR amplicon (MR1, MR2, MR2', and MR3) were cloned into the $\mathrm{XbaI}$-site of pUCm, respectively, forming the recombinant plasmids, pKUCm (5.8-kb), pKUCm1 (5.1-kb), pKUCm2 (4.8-kb), pKUCm2' (4.7-kb), and pKUCm3 (4.4-kb; Figure 1B).

\section{DETERMINATION OF THE FUNCTIONAL MINIMAL REPLICON AND TRANSFORMATION EFFICIENCY}

In order to verify the minimal replicon, the pKUCm series of vectors were introduced into $W$. confusa ATCC 10881 by electroporation, and transformants were selected using selective agar plates containing chloramphenicol. The electroporation efficiencies of pKUCm containing the entire pKW2124 plasmid and pKUCm1 were similar, suggesting that the MR1 region containing a putative ori region, RBS, and RepA should be required for optimum plasmid replication (Table 2 ). In addition, the electroporation efficiency of pKUCm2 was extremely low, even though the RBS and RepA were present in pKUCm2, indicating that the putative ori region is the key component for plasmid replication (Table 2). To confirm the role of RBS in Weissella plasmid replication, the vector pKUCm2' [pUCm+MR2' (only Rep protein 
Table 2 | Transformation efficiency of different pKUCm series vectors with the $W$. confusa ATCC 10881 host.

\begin{tabular}{ll}
\hline pKUCm series vector & $\begin{array}{l}\text { Transformation efficiency (CFU } \\
\boldsymbol{\mu g}^{-1} \text { DNA) }\end{array}$ \\
\hline pKUCm (Whole pKW2124) & $1.9 \times 10^{5}$ \\
pKUCm1 (ori+RBS+repA) & $1.5 \times 10^{5}$ \\
pKUCm2 (RBS+repA) & $1.3 \times 10^{1}$ \\
pKUCm2' (repA) & No colony \\
pKUCm3 (disrupted repA) & No colony \\
\hline
\end{tabular}

without RBS)] was constructed with PCR-amplified MR2' region with a forward primer (WC-F2'-XbaI) and a reverse primer (WCR1-XbaI; Table 1). This constructed vector, pKUCm2' vector was transformed into Weissella host. However, no colony was detected even after 3 days incubation at $37^{\circ} \mathrm{C}$, suggesting that RBS is an essential component for Weissella plasmid replication. The electroporation of pKUCm3 into W. confusa host showed no transformants, probably due to a lack of components required for plasmid replication (Table 2). Based on the electroporation results, the MR1 region is the minimum replicon of plasmid pKW2124. This minimal MR1 replicon was used for further construction of a Weissella expression vector with optimum transformation efficiency.

In this study, the transformation efficiency of the pKUCm series of vectors showed that $\mathrm{pKUCm}$ vectors containing the complete MR1 region (pKUCm and $\mathrm{pKUCm} 1$ ) were easily used to stably transform hosts via electroporation. However, the pKUCm vectors containing a partial MR1 region or lacking the MR1 region (pKUCm2 and pKUCm3, respectively) were highly unstable or did not replicate in the host. While these transformation efficiencies were studied with the vectors in E. coli, we confirmed these pKUCm series vector transformation efficiencies between Weissella hosts using vectors isolated from Weissella transformants to re-transform Weissella hosts. Interestingly, the re-transformation efficiencies increased (Figure 2). In particular, the re-transformation efficiency of pKUCm2, which showed very low transformation efficiency from E. coli to Weissella, was strikingly increased by more than $10^{3}$ times. While the reason for improvement of transformation efficiency during retransformation between Weissella hosts is not clearly understood at this time, it may be due to the restriction-modification (R-M) system of the Weissella host.

To confirm MR1 as a minimal plasmid replicon, the MR1 and CAT gene were obtained from pKUCm1 vector using PCR with a forward primer (pMR1-F-PstI) and a reverse primer (pMR1-RPstI). The PCR product was digested with PstI and self-ligated, resulting in a $2.7-\mathrm{kb}$ pMR1 (Table 1). The vector pMR1 was electroporated into $W$. confusa host, showing a transformation efficiency of $3.6 \times 10^{3} \mathrm{CFU} / \mu \mathrm{g}$ DNA, substantiating that MR1 is a Weissella plasmid minimal replicon. Interestingly, subsequent electroporation of pMR1 after recovery from the selected W. confusa transformant showed approximately 67 -fold increment of its transformation efficiency $\left(2.4 \times 10^{5} \mathrm{CFU} / \mu \mathrm{g} \mathrm{DNA}\right)$, probably due to the host R-M system (Figure 2).

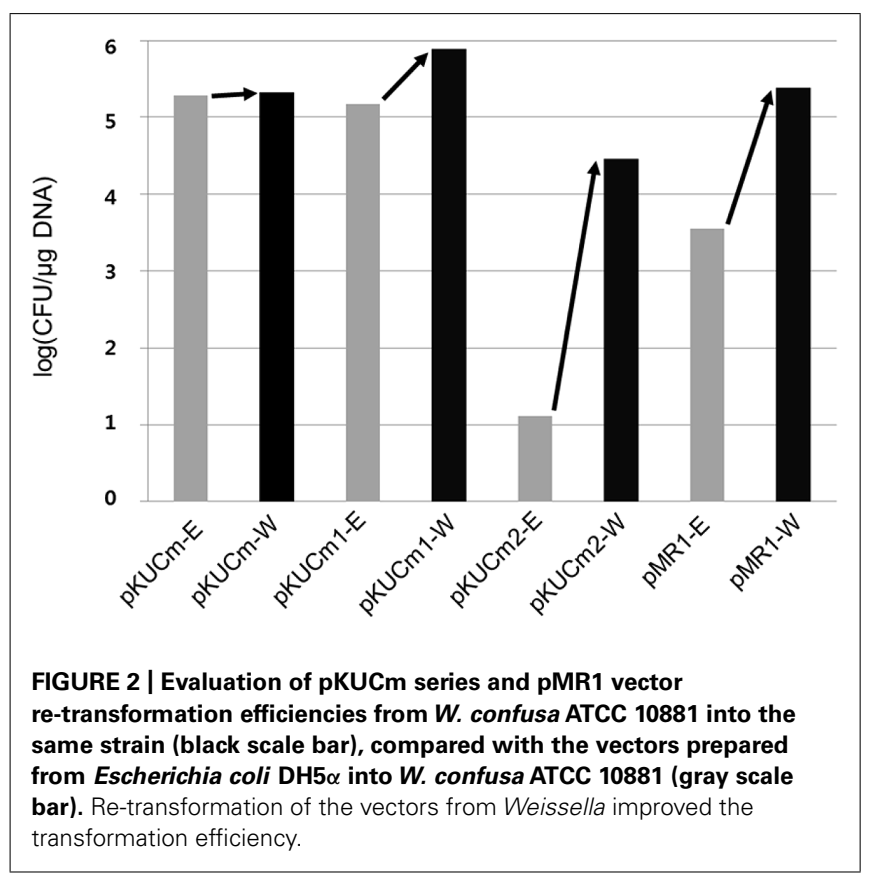

\section{SEGREGATIONAL PLASMID STABILITY}

The plasmid stability results of the pKUCm, pKUCm1, and pKUCm 2 vectors in their transformants are illustrated in Figure 3. In the absence of antibiotic selective pressure, nearly $100 \%$ of the transformants retained the $\mathrm{pKUCm}$ and $\mathrm{pKUCm} 1$ vectors for up to 100 generations, most likely because both of these vectors contain the MR1 region for optimum plasmid replication and plasmids containing the MR1 from pKW2124 are replicated by theta replication (Kim et al., 2013). Previous segregational plasmid stability results of pKW2124 also support this finding. However, plasmid stability results of pKUCm2 revealed that this vector is unstable in the transformant, most likely due to a lack of the putative ori region, resulting in the complete loss of the vector after 60 generations. This segregational plasmid stability test also

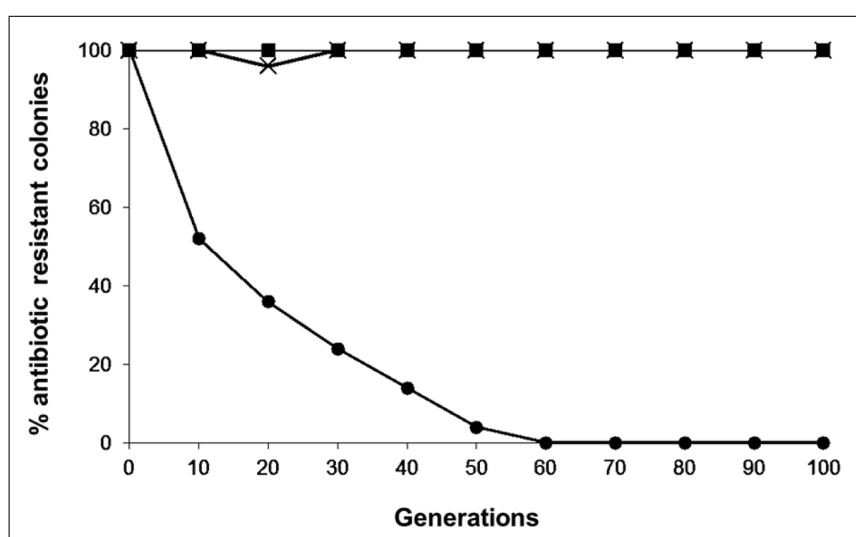

FIGURE 3 | Segregational stability of pKUCm, pKUCm1, and pKUCm2 in $\boldsymbol{W}$. confusa ATCC 10881. The presence of plasmids was verified by plasmid preparation and restriction digest analysis followed by gel electrophoresis. Symbols: pKUCm, (ם); pKUCm1, (X); pKUCm2, 
substantiated the fact that the MR1 region is required for optimal plasmid replication.

\section{HOST RANGE OF pKUCm1}

To investigate the replication ability of $\mathrm{pKUCm} 1$ in various host strains, the vector was introduced into the closely related LAB and Bifidobacterium strains listed in Table 1. The pKUCm1 plasmid was able to replicate in different genera, including Leuconostoc citreum, Lactococcus lactis, Lactobacillus plantarum, and W. paramesenteroides, although their transformation efficiencies were very low (Table 3), and pKUCm 1 could not replicate in the Streptococcus thermophilus and Bifidobacterium bifidum species (Table 3 ). Subsequent transformation efficiencies of $\mathrm{pKUCm} 1$ vector recovered from each selected LAB transformant into the same host competent cells were not quite different from the first transformation efficiencies, but slightly increased, supporting the predicted contribution of plasmid transformation efficiency and stability by the host R-M system (Table 3). This result suggests that pKUCm1 is a broad-host range vector and may be able to be used as a molecular tool in LAB.

\section{ANALYSIS OF THE $\boldsymbol{W}$. cibaria KSD1 $\beta$-GALACTOSIDASE GENE AND PROMOTER REGION}

To verify that the pKUCm1 can be used as a gene expression vector, we cloned and expressed the $\beta$-galactosidase gene. The DNA sequence of the lac $Z$ gene encoding $\beta$-galactosidase was obtained from the genome sequence of $W$. cibaria KSD1 (Jung et al., Unpublished data). The $W$. cibaria KSD1 $\beta$-galactosidase was encoded by two overlapping genes, a large subunit gene (1,896-bp encoding 636 amino acids) and a small subunit gene (951-bp encoding 316 amino acids). The large subunit gene is located upstream of the small subunit gene and the two genes overlap by 20 bp nucleotides (Figure 4). The putative -35 and -10 regions were predicted using the BPROM program (Li, 2011), which are located approximately 200-bp upstream of the start codon for the

Table 3 | Transformation efficiency of pKUCm1 with selected LAB and B. bifidum hosts.

\begin{tabular}{lll}
\hline Bacterial strain & \multicolumn{2}{c}{$\begin{array}{c}\text { Transformation efficiency (CFU } \\
\boldsymbol{\mu \mathbf { g } ^ { - 1 }} \mathbf{~ D N A )}\end{array}$} \\
\cline { 2 - 3 } & $\begin{array}{l}\text { First } \\
\text { transformation }\end{array}$ & $\begin{array}{l}\text { Second } \\
\text { transformation }\end{array}$ \\
\hline Leuconostoc citreum 75X & $2.7 \times 10^{0}$ & $3.7 \times 10^{0}$ \\
Lactococcus lactis MG1363 & $1.3 \times 10^{0}$ & $1.3 \times 10^{0}$ \\
Lactobacillus plantarum 50W & $1.0 \times 10^{0}$ & $1.7 \times 10^{0}$ \\
W. paramesenteroides KCTC 3531 & $5.3 \times 10^{0}$ & $7.7 \times 10^{0}$ \\
Streptococcus thermophilus ATCC & No colonies & - \\
19258 & & - \\
B. bifidum ATCC 15521 & No colonies & - \\
\hline
\end{tabular}

a First transformation indicates the transformation of $p K U \mathrm{Cm} 1$ from the selected E. coli transformant into each $L A B$ competent cell; Second transformation indicates the re-transformation of $p K U C m 1$ from each selected $L A B$ transformant into the same host competent cell.

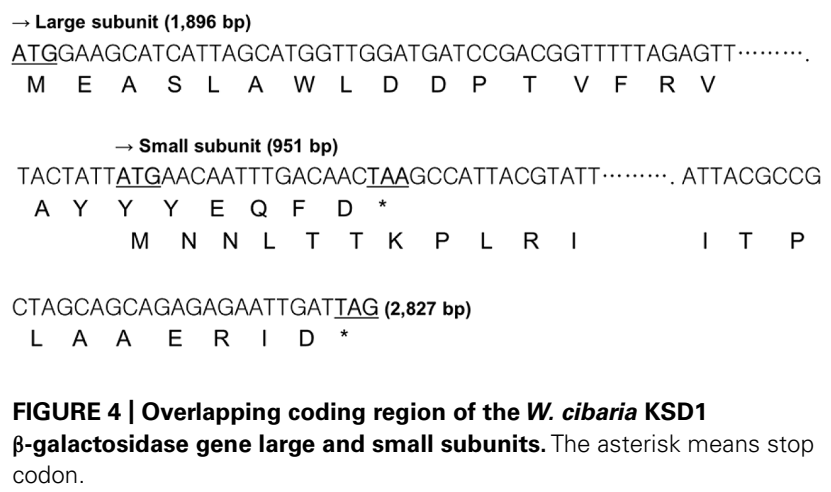

FIGURE 4 | Overlapping coding region of the $W$. cibaria KSD1 $\beta$-galactosidase gene large and small subunits. The asterisk means stop codon.

large subunit (Figure 5). Based on these results, two forward and reverse primers, WC-proGalF-BamHI and WC-proGalR-BamHI, were chemically synthesized for PCR amplification of the $W$. cibaria KSD1 $\beta$-galactosidase gene containing its promoter region (Table 1).

\section{$\beta$-GALACTOSIDASE GENE EXPRESSION USING pKUCm1 AND DETERMINATION OF ENZYME ACTIVITY}

The $W$. cibaria KSD1 $\beta$-galactosidase gene containing its native promoter region was PCR-amplified to generate a 3.1-kb PCR product. The PCR product was purified and cloned into the BamHI site of pKUCm1, forming the recombinant plasmid pKUGal (Figure 6), which was then introduced into $W$. confusa ATCC 10881 by electroporation. Selected transformants containing pKUGal were confirmed by plasmid re-isolation and restriction enzyme digestion analysis (Figure 7 ). In addition, $\beta$-galactosidase production by the selected transformant harboring pKUGal was confirmed using the blue-white screening method. After incubation of the selected transformant on MRS agar containing X-gal and IPTG, blue colonies were isolated while the wild-type $W$. confusa ATCC 10881 did not show blue colonies (Figure 8). To determine the enzyme activity of $\beta$-galactosidase, the selected transformant containing pKUGal and the wild-type $W$. confusa ATCC 10881 negative control were incubated for $10 \mathrm{~h}$. After incubation and cell disruption, the supernatant of the negative control did not show any enzyme activity for lactose utilization, while the supernatant of the selected transformant showed an enzyme activity of $0.34 \mathrm{U} / \mathrm{ml}$,

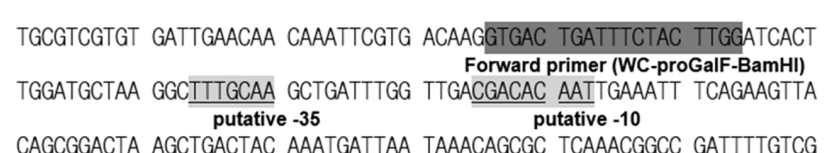

GTACGgTtTG GGCGCTGTTT TGGTATTAAG TAACGAATCG TCATAAGACG CCGGGTGGTT

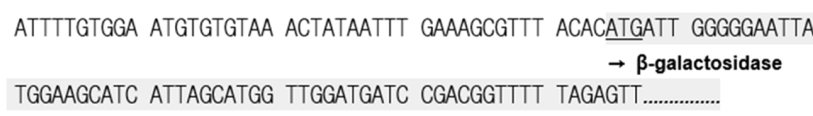

FIGURE 5 | Putative promoter region of the $\boldsymbol{W}$. cibaria KSD1 $\boldsymbol{\beta}$-galactosidase gene. The promoter region was predicted using the BPROM program. 


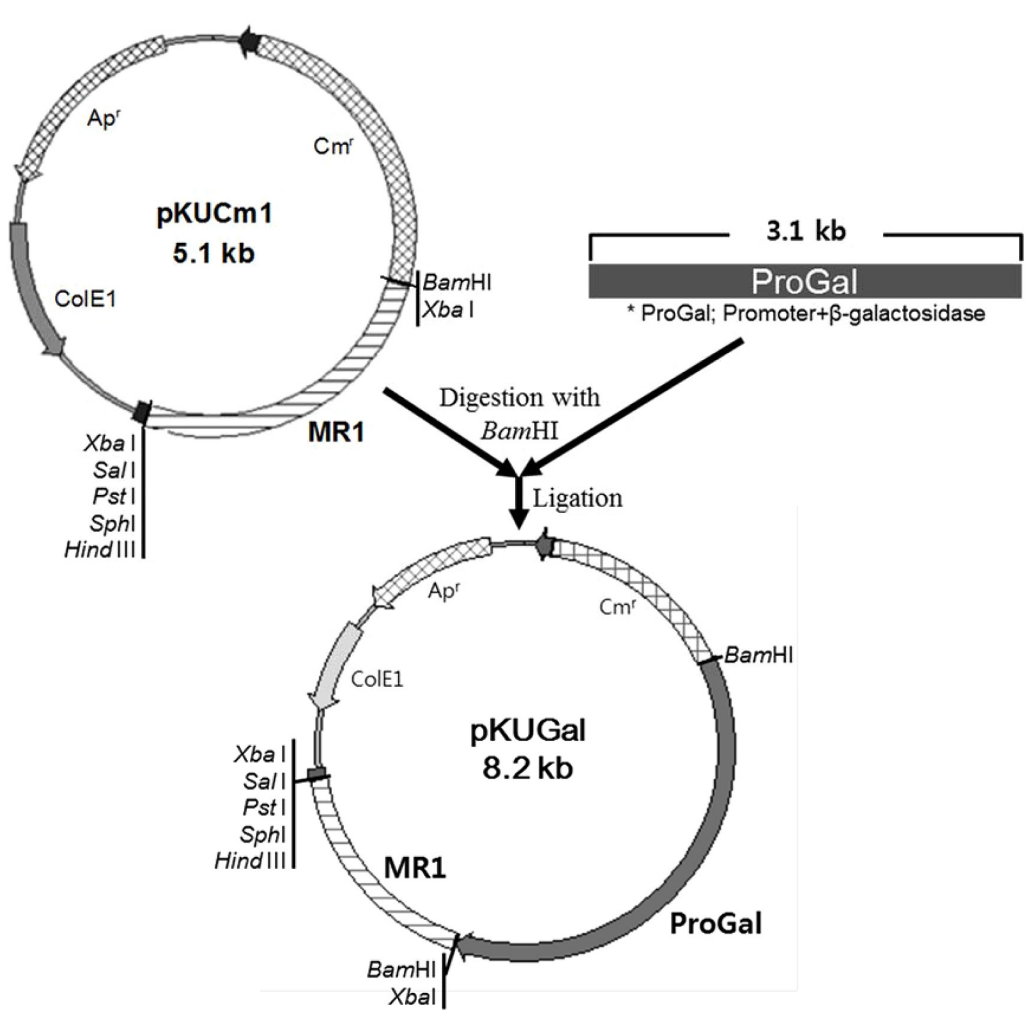

FIGURE 6 | Construction of the cloned pKUGal vector for $\boldsymbol{\beta}$-galactosidase gene expression. The $\beta$-galactosidase gene containing the putative promoter region (ProGal) was cloned into the BamHI site of pKUCm1.

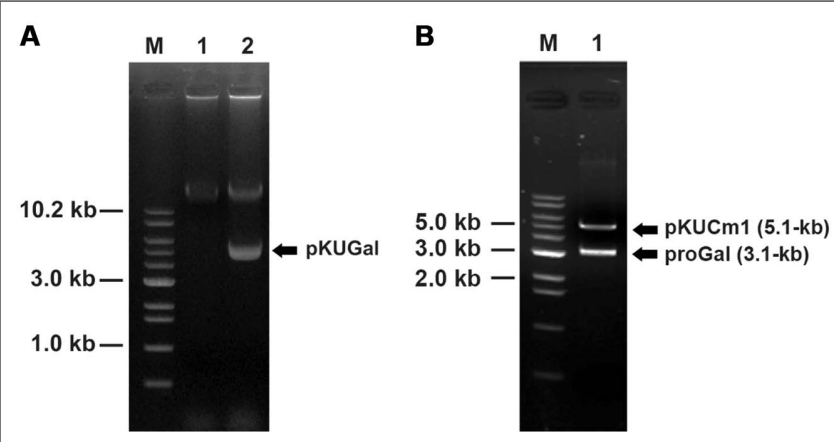

FIGURE 7 | Confirmation of vector introduction into $W$. confusa ATCC 10881 using restriction analyses followed by gel electrophoresis.

(A) Lane 1, Wild-type W. confusa ATCC 10881; Lane 2, W. confusa ATCC 10881 harboring pKUGal; M, 1.0 kb DNA ladder (Bioneer). (B) gel-extracted pKUGal (8.2-kb) digested to 5.1-kb pKUCm1 and 3.1-kb ProGal by BamHI.

confirming that the selected transformant produced intracellular $\beta$-galactosidase.

\section{DISCUSSION}

The genus Weissella has been frequently detected and isolated from various fermented foods worldwide (except for W. soli that was isolated from a soil sample; Magnusson et al., 2002; Lee et al., 2010), indicating that Weissella species may play an important role in food

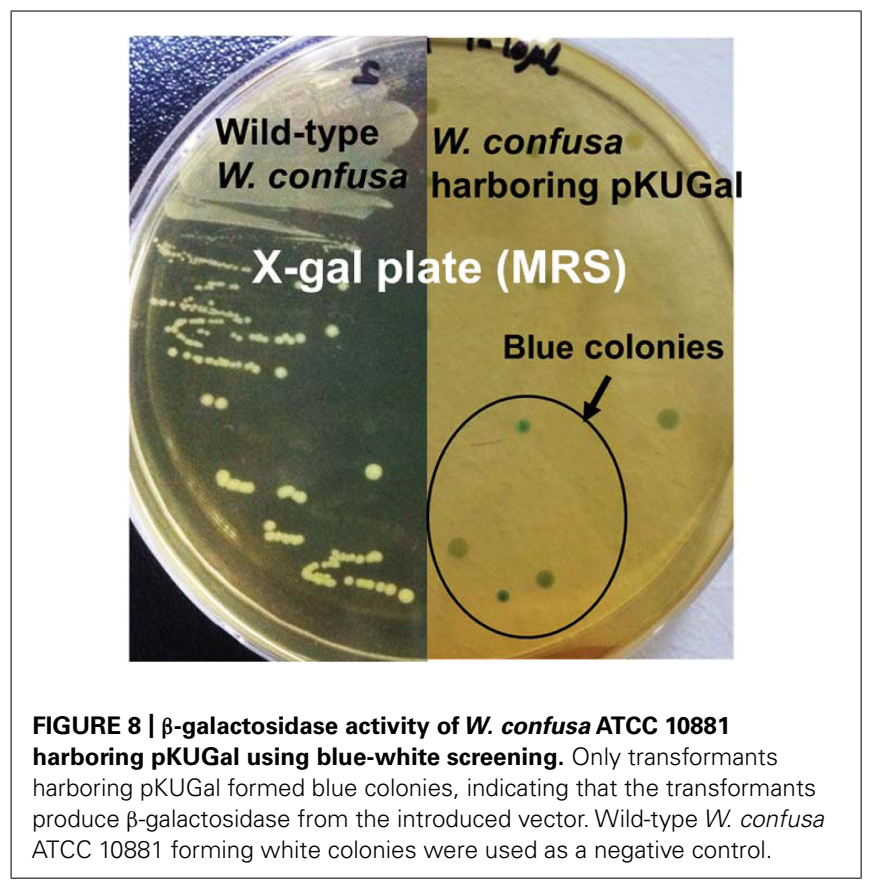

fermentation. The Weissella genus has been recognized as a potential probiotic due to its frequent detection and isolation in foods; however, Fairfax (2014) recently suggested that W. confusa may 
be linked to infectious diseases in humans and animals, potentially causing sepsis and other serious infections, implying that use of $W$. confusa may need careful consideration for probiotic applications. Nevertheless, the probiotic study of various Weissella species has focused on human health-promoting properties such as antimicrobial activity against pathogens (Cai et al., 1998), inhibition of adherence of $H$. pylori (Nam et al., 2002), and reduction of volatile sulfur compound (VSC) production involved in the oral cavity (Kang et al., 2006). In particular, this genus is one of the most dominant microbes in traditionally Korean fermented foods including kimchi, makgeolli, and jeotgal, to name a few (Kim and Chun, 2005; Guan et al., 2011; Min et al., 2012). Molecular studies will need to be done to explore the health promoting effects of the genus Weissella.

To date, only four Weissella plasmids including pKLCA, pKLCB, pKLCC, and pKW2124 have been isolated and studied at the molecular level (Park etal., 2007; Kim et al., 2013). Among them, pKW2124 was replicated using the theta replication mechanism and is extremely stable in selected transformants (Kim et al., 2013). Therefore, this plasmid was used for the construction of a cloning and expression vector, pKWCSLGFP (8.6-kb), which was tested by the cloning and expression of the $g f p$ gene. This vector successfully expressed and produced GFP protein as a surface display vector in the genus Weissella (Kim et al., 2013). However, this vector required optimization by minimizing the Weissella plasmid pKW2124 replicon for easy handling and better transformation efficiency. To determine the minimal replicon of the Weissella plasmid, four different plasmid regions (MR1, MR2, MR2', and MR3) were obtained by PCR amplification. After cloning and transformation of pKUCm (whole plasmid pKW2124), pKUCm1 (MR1), pKUCm2 (MR2), pKUCm2' (MR2'), and pKUCm3 (MR3), the minimal replicon of pKW2124 was determined to be a putative ori region, an RBS, and the repA gene as essential components for plasmid replication. Previous studies that minimized plasmid replicons in other genera including Leuconostoc, Bifidobacterium, and Lactobacillus, demonstrated that the minimal replicons consist of these same three essential components (Cronin et al., 2007; Yin et al., 2009; Chae et al., 2013). Furthermore, transformation efficiencies of pKUCm and pKUCm1 were not different, substantiating the fact that the MR1 region contains the minimal replicon for pKW2124 replication. Using the MR1 region, the minimized cloning vector $\mathrm{pKUCm} 1$ was further used for specific gene expression.

Actually, the vector pKUCm1 contains two replicons, ColE1 from pUC19 and Weissella plasmid replicon from pKW2124. Therefore, it may be important to clarify which plasmid replicon really works for Weissella host. The additional experiment to clarify this, the vector pUCm without Weissella plasmid replicon was chosen and electroporated into W. confusa. However, no colony was detected on the selective agar plates containing chloramphenicol, substantiating that the ColE1 replicon in pUCm vector does not work for plasmid replication in Weissella host (data not shown). In addition, the results of pUCm (pUC19+CmR), pKUCm2 [pUCm+MR2 (without plasmid ori of Weissella)], pKUCm3 [pUCm+MR3 (with only fragmented Rep protein)] in Table 2 were disappointing for cloned vector replications in Weissella, substantiating that only ColE1 replicon cannot replicate the vector replication. It was previously reported that the ColE1 replicon is one of the narrow-host-range replicons like pSC101 (Kües and Stahl, 1989) and it requires only replication proteins from E. coli host (Tomizawa et al., 1977; Donoghue and Sharp, 1978). To confirm this, Diaz and Staudenbauer (1982) showed that ColE1 replicon cannot replicate in cell-free Pseudomonas extracts but it showed a partial replication ability when purified E. coli gyrase and DNA polymerase I are added, substantiating this narrow host range of ColE1 replicon. However, ColE1 replicon in pKUCm1 may still need to make cloning of specific gene easier in E. coli host than in Weissella host. To verify the contribution of the ColE1 replicon for plasmid replication in Weissella, only $\mathrm{Cm}^{\mathrm{r}}$ and Weissella minimal replicon regions in pKUCm1 vector were amplified by PCR and self-ligated. And then the ligated plasmid, designated 2.7-kb pMR1, was electroporated into the Weissella host strain. Additional transformation experiment into $W$. confusa host strain with pMR1 showed lower transformation efficiency $\left(3.6 \times 10^{3} \mathrm{CFU} / \mu \mathrm{g} \mathrm{DNA}\right)$ than that of $\mathrm{pKUCm} 1$ vector $\left(1.5 \times 10^{5}\right.$ $\mathrm{CFU} / \mu \mathrm{g}$ DNA), indicating that the elements of ColE1 replicon may contribute to replication of pKUCm1 vector in Weissella. After recovery of pMR1 from the selected $W$. confusa transformant, it was re-transformed into the $W$. confusa competent cells, showing the increment of transformation efficiency of pMR1 in Weissella $\left(2.4 \times 10^{5} \mathrm{CFU} / \mu \mathrm{g}\right.$ DNA $)$. However, this enhanced transformation efficiency of pMR1 is still slightly lower than that of re-transformed pKUCm1 vector $\left(7.8 \times 10^{5} \mathrm{CFU} / \mu \mathrm{g}\right.$ DNA), suggesting that contribution of the ColE1 replicon to the plasmid replication in Weissella may be still valid even in the re-transformation into Weissella, even though the host R-M system may be involved in this increment of the transformation efficiency.

For expression of lac $Z$ gene, the putative promoter region (TTGTGG as putative -35 box and TATAAT as putative -10 box) was initially found upstream lac $Z$ gene of $W$. cibaria KSD1. To amplify this lac $Z$ gene containing the putative promoter region using $\mathrm{PCR}$, a primer set including a forward primer (5'-GGATCCCCGGGTGGTTATTTTGTGGA-3') and a reverse primer (WC-proGalR-BamHI in Table 1) was designed and chemically synthesized. This forward primer contains putative -35 box (TTGTGG, indicated in the forward primer with underbar). The insert DNA containing lacZ gene and its putative promoter region was obtained by PCR with this primer set and cloned into pKUCm1 expression vector. However, there was no blue colony on selective $\mathrm{X}$-gal plate, indicating that this gene was not expressed in the vector system, probably due to wrong promoter region. To detect the other candidate of consensus sequences as -35 and -10 boxes, the BPROM program was used for prediction of bacterial promoters $(\mathrm{Li}, 2011)$ and the other possible promoter region was detected (Figure 5). And then, the Weissella lacZ gene including this newly detected promoter region was amplified by PCR and cloned into the expression vector $\mathrm{pKUCm} 1$, designated $\mathrm{pKU}$ Gal. Finally, we detected blue colonies on selective X-gal plate, substantiating that newly detected promoter region really works for binding of ribosome to translate mRNA of $\beta$-galactosidase (Figure 8). 
In the previous study, the surface display vector pKWCSLGFP was constructed using pKW2124 and was used for $g f p$ gene expression (Kim et al., 2013). The advantage of the pKWCSLGFP vector is due to specific gene expression and secretion of the produced protein. In this study, the novel expression vector $\mathrm{pKUCm} 1$ was constructed using the minimal pKW2124 replicon that can be used for the expression of the $\beta$-galactosidase gene for intracellular production. Based on these two vector systems, a new Weissella expression/secretion vector system combining the advantages of these two vectors could be developed as a future goal. Furthermore, strong promoters and inducible promoters need to be studied to improve levels of gene expression in this Weissella vector system. A version of pKUGal without its own promoter could be developed and used for promoter screening in Weissella.

This newly developed expression vector system showed high stability in the host strains as well as specific gene expression and protein production. Therefore, this vector system could be used for specific gene delivery/expression and production of protein in Weissella. In addition, this vector system may be useful for the elucidation of specific Weissella gene functions as a molecular genetic tool via cloning/expression or knockout of specific Weissella genes. This is the first report of a plasmid minimal replicon and its application for development of an improved vector system in Weissella.

\section{ACKNOWLEDGMENTS}

The Basic Science Research Program through the National Research Foundation of Korea (NRF) funded by the Ministry of Science, ICT and Future Planning (No, NRF-2012R1A1A1009859) supported this research. This research was also supported by the High Value-added Food Technology Development Program, Ministry for Food, Agriculture, Forestry and Fisheries, Republic of Korea (no. 312002033SB010).

\section{REFERENCES}

Amari, M., Laguerre, S., Vuillemin, M., Robert, H., Loux, V., Klopp, C., et al. (2012). Genome sequence of Weissella confusa LBAE C39-2, isolated from a wheat sourdough. J. Bacteriol. 194, 1608-1609. doi: 10.1128/JB.06788-11

Benomar, N., Abriouel, H., Lee, H., Cho, G. S., Huch, M., Pulido, R. P., et al. (2011). Genome sequence of Weissella thailandensis fsh4-2. J. Bacteriol. 193:5868. doi: 10.1128/JB.05883-11

Cai, Y., Benno, Y., Nakase, T., and Oh, T. K. (1998). Specific probiotic characterization of Weissella hellenica DS-12 isolated from flounder intestine. J. Gen. Appl. Microbiol. 44, 311-316. doi: 10.2323/jgam.44.311

Chae, H. S., Lee, S. H., Lee, J. H., Park, S. J., and Lee, P. C. (2013). Use of a novel Escherichia coli-Leuconostoc shuttle vector for metabolic engineering of Leuconostoc citreum to overproduce D-lactate. Appl. Environ. Microbiol. 79, 1428 1435. doi: 10.1128/AEM.03291-12

Collins, M. D., Samelis, J., Metaxopoulos, J., and Wallbanks, S. (1993). Taxonomic studies on some Leuconostoc-like organisms from fermented sausages: description of a new genus Weissella for the Leuconostoc paramesenteroides group of species. J. Appl. Bacteriol. 75, 595-603. doi: 10.1111/j.1365-2672.1993.tb01600.x

Cronin, M., Knobel, M., O'connell-Motherway, M., Fitzgerald, G. F., and Van Sinderen, D. (2007). Molecular dissection of a bifidobacterial replicon. Appl. Environ. Microbiol. 73, 7858-7866. doi: 10.1128/AEM.01630-07

Diaz, R., and Staudenbauer, W. L. (1982). Replication of the broad host range plasmid RSF1010 in cell-free extracts of Escherichia coli and Pseudomonas aeruginosa. Nucleic Acids Res. 10, 4687-4702. doi: 10.1093/nar/10.15.4687

Donoghue, D. J., and Sharp, P. A. (1978). Replication of colicin E1 plasmid DNA in vivo requires no plasmid-encoded proteins. J. Bacteriol. 133, 1287-1294.

Fairfax, M. R. (2014). Weissella confusa: problems with identification of an opportunistic pathogen that has been found in fermented foods and proposed as a probiotic. Food Microbiol. 5, 254-258. doi: 10.3389/fmicb.2014. 00254

Galle, S., Schwab, C., Arendt, E., and Ganzle, M. (2010). Exopolysaccharide-forming Weissella strains as starter cultures for sorghum and wheat sourdoughs. J. Agric. Food Chem. 58, 5834-5841. doi: 10.1021/jf1002683

Garrity, G. M., Lilburn, T. G., Cole, J. R., Harrison, S. H., Euzeby, J., and Tindall, B. J. (2007). Introduction to the Taxonomic Outline of Bacteria and Archaea (TOBA) Release 7.7. The Taxonomic Outline of Bacteria and Archaea 7, 1-5. Available at: http://www.taxonomicoutline.org/index.php/toba/issue/current

Guan, L., Cho, K. H., and Lee, J.-H. (2011). Analysis of the cultivable bacterial community in jeotgal, a Korean salted and fermented seafood, and identification of its dominant bacteria. Food Microbiol. 28, 101-113. doi: 10.1016/j.fm.2010. 09.001

Horinouchi, S., and Weisblum, B. (1982). Nucleotide sequence and functional map of pC194, a plasmid that specifies inducible chloramphenicol resistance. J. Bacteriol. 150, 815-825.

Kang, M. S., Kim, B. G., Chung, J., Lee, H. C., and Oh, J. S. (2006). Inhibitory effect of Weissella cibaria isolates on the production of volatile sulphur compounds. J. Clin. Periodontol. 33, 226-232. doi: 10.1111/j.1600-051X.2006. 00893.x

Kim, D. S., Choi, S. H., Kim, D. W., Nam, S. H., Kim, R. N., Kang, A., et al. (2011). Genome sequence of Weissella cibaria KACC 11862. J. Bacteriol. 193, 797-798. doi: 10.1128/JB.01342-10

Kim, M., and Chun, J. (2005). Bacterial community structure in kimchi, a Korean fermented vegetable food, as revealed by $16 \mathrm{~S}$ rRNA gene analysis. Int. J. Food Microbiol. 103, 91-96. doi: 10.1016/j.ijfoodmicro.2004.11.030

Kim, S. Y., Oh, C. G., Lee, Y. J., Choi, K. H., Shin, D. S., Lee, S. K., et al. (2013). Sequence analysis of a cryptic plasmid pKW2124 from Weissella cibaria KLC140 and construction of a surface display vector. J. Microbiol. Biotechnol. 23, 545-554. doi: 10.4014/jmb.1301.01018

Kües, U., and Stahl, U. (1989). Replication of plasmids in gram-negative bacteria. Microbiol. Rev. 53, 491-516.

Lee, J. H., Bae, J. W., and Chun, J. (2012). Draft genome sequence of Weissella koreensis KCTC 3621T. J. Bacteriol. 194, 5711-5712. doi: 10.1128/JB. 01356-12

Lee, J. H., and O'Sullivan, D. J. (2006). Sequence analysis of two cryptic plasmids from Bifidobacterium longum DJO10A and construction of a shuttle cloning vector. Appl. Environ. Microbiol. 72, 527-535. doi: 10.1128/AEM.72.1.527535.2006

Lee, J. S., Lee, K. C., Ahn, J. S., Mheen, T. I., Pyun, Y. R., and Park, Y. H. (2002). Weissella koreensis sp. nov., isolated from kimchi. Int. J. Syst. Evol. Microbiol. 52, 1257-1261. doi: 10.1099/ijs.0.02074-0

Lee, K. W., Park, J. Y., Chun, J., Han, N. S., and Kim, J. H. (2010). Importance of Weissella species during kimchi fermentation and future works. Kor. J. Microbiol. Biotechnol. 38, 341-348.

Lee, S. H., Jung, J. Y., Lee, S. H., and Jeon, C. O. (2011). Complete genome sequence of Weissella koreensis KACC 15510, isolated from kimchi. J. Bacteriol. 193:5534. doi: 10.1128/JB.05704-11

Li, R. W. (2011). Metagenomics and its Applications in Agriculture, Biomedicine, and Environmental Studies. New York, NY: Nova Science Publisher's.

Magnusson, J., Jonsson, H., Schnurer, J., and Roos, S. (2002). Weissella soli sp. nov., a lactic acid bacterium isolated from soil. Int. J. Syst. Evol. Microbiol. 52, 831-834. doi: 10.1099/ijs.0.02015-0

Masuda, Y., Zendo, T., Sawa, N., Perez, R. H., Nakayama, J., and Sonomoto, K. (2012). Characterization and identification of weissellicin $Y$ and weissellicin $M$, novel bacteriocins produced by Weissella hellenica QU 13. J. Appl. Microbiol. 112, 99-108. doi: 10.1111/j.1365-2672.2011.05180.x

Miller, J. H. (1972). Experiments in Molecular Genetics. New York, NY: Cold Spring Harbor Laboratory Press.

Min, J., Nam, Y., Ju, J., Jung, J., Lee, J., and Kim, H. (2012). Changes of yeast and bacterial flora during fermentation and storage of Gugija-Liriope tuber Makgeolli using PCR-DGGE. Kor. J. Microbiol. Biotechnol. 40, 111-116. doi: 10.4014/kjmb.1202.02004

Nam, H., Ha, M., Bae, O., and Lee, Y. (2002). Effect of Weissella confusa strain PL9001 on the adherence and growth of Helicobacter pylori. Appl. Environ. Microbiol. 68, 4642-4645. doi: 10.1128/AEM.68.9.4642-4645.2002

O'Sullivan, D. J., and Klaenhammer, T. R. (1993). Rapid mini-prep isolation of high-quality plasmid DNA from Lactococcus and Lactobacillus spp. Appl. Environ. Microbiol. 59, 2730-2733. 
Park, M. S., Kim, S. H., Kim, J. D., Oh, S. E., Park, K. J., Lee, S. K., et al. (2007). Molecular characterization of plasmid DNA from Weisselia cibaria isolated from kimchi. Kor. J. Genet. 29, 29-35.

Sambrook, J., Fritsch, E. F., and Maniatis, T. (2001). Molecular Cloning: A Laboratory Manual. New York, NY: Cold Spring Harbor Laboratory Press.

Srionnual, S., Yanagida, F., Lin, L. H., Hsiao, K. N., and Chen, Y. S. (2007). Weissellicin 110, a newly discovered bacteriocin from Weissella cibaria 110, isolated from plaa-som, a fermented fish product from Thailand. Appl. Environ. Microbiol. 73, 2247-2250. doi: 10.1128/AEM.02484-06

Tohno, M., Kitahara, M., Inoue, H., Uegaki, R., Irisawa, T., Ohkuma, M., et al. (2013). Weissella oryzae sp. nov., isolated from fermented rice grains. Int. J. Syst. Evol. Microbiol. 63, 1417-1420. doi: 10.1099/ijs.0.043612-0

Tomizawa, J. I., Ohmori, H., and Bird, R. E. (1977). Origin of replication of colicin E1 plasmid DNA. Proc. Natl. Acad. Sci. U.S.A. 74, 1865-1869. doi: 10.1073/pnas.74.5.1865

Wegmann, U., O'connell-Motherway, M., Zomer, A., Buist, G., Shearman, C., Canchaya, C., et al. (2007). Complete genome sequence of the prototype lactic acid bacterium Lactococcus lactis subsp. cremoris MG1363. J. Bacteriol. 189, 3256-3270. doi: 10.1128/JB.01768-06

Yin, S., Hao, Y., Zhai, Z., Zhang, W., Zhou, H., Wang, G., et al. (2009). Functional analysis of the plasmid pM4 replicon from Lactobacillus plantarum M4: determination of the minimal replicon and functionality identification of the putative sso. Plasmid 62, 166-171. doi: 10.1016/j.plasmid.2009.07.004

Conflict of Interest Statement: The authors declare that the research was conducted in the absence of any commercial or financial relationships that could be construed as a potential conflict of interest.

Received: 17 October 2014; accepted: 12 January 2015; published online: 03 February 2015.

Citation: Ku H-J, Park MS and Lee J-H (2015) Characterization of a minimal pKW2124 replicon from Weissella cibaria KLC140 and its application for the construction of the Weissella expression vector pKUCm1. Front. Microbiol. 6:35. doi: 10.3389/fmicb.2015.00035

This article was submitted to Food Microbiology, a section of the journal Frontiers in Microbiology.

Copyright (C) $2015 \mathrm{Ku}$, Park and Lee. This is an open-access article distributed under the terms of the Creative Commons Attribution License (CC BY). The use, distribution or reproduction in other forums is permitted, provided the original author(s) or licensor are credited and that the original publication in this journal is cited, in accordance with accepted academic practice. No use, distribution or reproduction is permitted which does not comply with these terms. 